\title{
Estrategias de intervención institucional
}

Mag. María Inés Vázquez*

\begin{abstract}
Este artículo tiene como propósito compartir algunas reflexiones surgidas de la investigación, en torno a la convivencia de dos modelos de gestión en educación media pública -uno vigente y otro en construcción- promovidos por el denominado Plan 96. Surgen del análisis ciertos "ejes de tensión", cuya resolución podría transformar potenciales amenazas en oportunidades para consolidar los procesos de transformación puestos en marcha. Asimismo se plantean posibles estrategias para fortalecer la gestión, a partir de un abordaje global e integrador.
\end{abstract}

Los modelos de análisis institucional han tenido una evolución significativa a partir de la segunda mitad del siglo XX y especialmente a partir de la década de los 90 . Así por ejemplo, en la década de los 50 situamos la visión normativa, que se ha caracterizado por un abordaje racional de la realidad institucional, basado en manuales de procedimientos. Esta forma simple de entender la organización ha evolucionado hacia otros modelos mucho más sofisticados, que jerarquizan a las personas como factor activo de los procesos de transformación, cambio o mejora, por ser quienes en definitiva habilitan o dificultan su consolidación. A este fenómeno se lo denomina "emergencia del sujeto", Casassus (2002).

Estos modelos de análisis, permiten presentar en forma sencilla situaciones que en la realidad se caracterizan por su dinámica y complejidad; representan una abstracción que incorpora y jerarquiza algunos aspectos, dejando otros en un segundo plano, siempre respaldando ese análisis selectivo con un cierto marco teórico.

Los nuevos modelos incorporan aspectos que tienen que ver con lo individual, con lo grupal, con el contexto organizacional, habiendo contribuido a que las propuestas de transformación institucional también resulten de mayor complejidad en su implementación, y requieran de ciertas habilidades y competencias para liderarlas. Es entonces cuando los conceptos de gestión e intervención institucional adquieren especial relevancia.

Al decir de Hargreaves (2001) "las nuevas propuestas de cambio más integrales en su estructuración, también resultan más complejas en su implementación, incluso para los mejores docentes". Y agrega, que más allá del saber y la idoneidad de los profesionales involucrados, se requieren, en todos los casos, apoyos específicos.

Partiremos entonces del concepto de gestión institucional, como la capacidad no sólo de administrar sino también de dirigir, de promover, de integrar procesos educativos en escenarios caracterizados por el cambio y la incertidumbre.

En torno a intervención institucional, Lidia Fernández (1999) la define como una práctica psicosocial destinada a colaborar en la superación de dificultades presentes en la organización. Por su parte Cassasus (2002), afirma que las prácticas de intervención permiten optimizar la relación entre sistemas, estructuras, estrategias, estilos, capacidades personales, colectivas e institucionales. 
Estos desafíos requieren sin duda de gestores institucionales que desarrollen un liderazgo integral que articule lo pedagógico, administrativo-organizacional, político y comunitario. De alguna forma la gestión educativa, los enfrenta a la necesidad de aunar distintos niveles y requerimientos de la realidad, que refieren tanto a aspectos procedimentales como estructurales. No debemos perder de vista, que aquellas instituciones que forman parte de un sistema, deben establecer y consolidar mecanismos de articulación, que faciliten la coordinación y los acuerdos entre los distintos sectores implicados.

\section{La realidad como escenario de análisis}

Con el propósito de poner en evidencia este "entramado de relaciones", nos interesa compartir algunos aspectos de una producción en la que hemos estado trabajando, relacionada con la educación media pública.

Se trata de una investigación cuyo propósito fue avanzar en la comprensión y el análisis de los procesos de transformación a partir de una experiencia concreta: la implementación del Plan 96 en los liceos del ciclo básico, tomando como principal insumo la percepción de los involucrados.

Se trabajó sobre la base de la opinión de "informantes clave", que en esta oportunidad fueron: (i) las autoridades del Consejo de Educación Secundaria, (ii) la jefatura del Departamento de Recursos Humanos, (iii) la jefatura del Departamento Docente, (iv) Directores que se encuentran trabajando con el Plan 96 desde sus inicios, (v) Inspectores y Técnicos que han venido acompañando a estas instituciones.

Las técnicas que se utilizaron para el relevamiento de la información fueron: entrevistas semi-estructuradas y análisis de documentos.

De los resultados del estudio, nos interesa en este caso jerarquizar:

\section{(i) la convivencia de dos modelos}

Uno que denominaremos "top-down", Braslavsky (2001) y otro que llamaremos "interactivo", Marchesi y Martín (1998).

Se visualiza cierta dicotomía en la percepción que los diferentes actores educativos manifiestan en torno del proceso. Mientras que por una parte, prevalece la visión jerárquica que define estilos de implementación rígidos y verticales (Modelo top-down), por otra se pretenden consolidar procesos transversales que generen espacios de trabajo colectivo (Modelo interactivo).

(ii) la presencia de ciertos "ejes de tensión" o puntos de clivaje en la matriz cultural que sustenta estos dos modelos. La presencia de ciertos ejes de tensión entre ambos modelos uno vigente y otro en construcción - representa a nuestro entender, un momento histórico ideal para que el cambio efectivamente se produzca, siempre y cuando: a) puedan ser visualizados estos ejes de tensión y b) puedan ser trabajados en forma adecuada. Si esto se logra, se vuelven viables las transformaciones proyectadas de los diferentes niveles implicados. De lo contrario, es posible que se vuelva en el corto o el mediano plazo a situaciones institucionales anteriores. 
Estos ejes de tensión detectados, refieren a aspectos específicos (ver el diagrama) a saber:

Formas de organización: la tensión se produce ante la convivencia de procesos netamente administrativos y otros basados en la lógica de proyectos. Así por ejemplo administrativamente se sigue contratando al docente por horas de clase, mientras que desde el nuevo modelo se espera que el mismo participe en un proyecto donde su mirada, su interés, su esfuerzo no estén puestos exclusivamente en el aula, sino en la realidad institucional en su conjunto.

Formas de vinculación: mientras que desde el modelo top-down las propuestas tienden a "bajar" desde las oficinas centrales, se promueven en el ámbito de los centros otras formas de vinculación en red, que guardan relación con la herramienta proyecto, donde las interacciones que se intentan promover refieren a equipos de trabajo.

Vías de comunicación: mientras que en el modelo tradicional las vías son burocráticas y el expediente es "rey y señor", desde el nuevo modelo se intentan promover vínculos directos y personalizados.

Estilos de conducción: mucho más directivo en uno y mucho más interactivo en el otro, en el cual la capacidad de apoyar, orientar, promover el trabajo colegiado adquiere especial relevancia. El liderazgo en este último caso, pone énfasis no sólo en la realización de la tarea, sino también en las transacciones que se establecen para cumplirla.

Incorporación tecnológica: si bien a nivel de política educativa se detecta una fuerte apuesta a la informatización del sistema, desde el punto de vista lo que se constata en muchos casos, es el uso de esta tecnología como prolongación del lápiz, Brunner (2000).

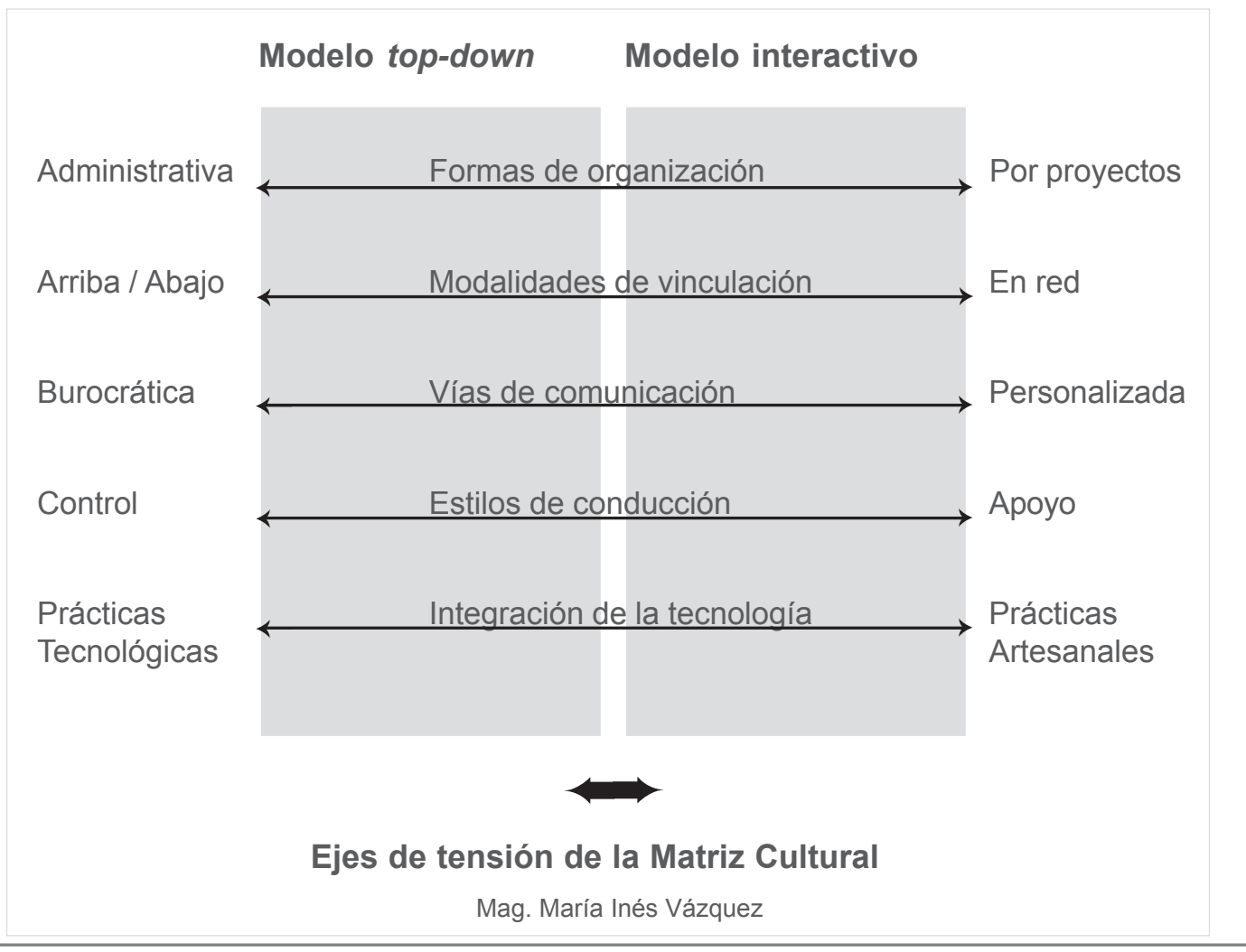


Este panorama que acabamos de esbozar nos recuerda una frase de Hargreaves (2001) quien dice "la gramática escolar hace que muchas veces los actores institucionales queden atrapados en la propia estructura organizativa, más que en las propuestas de cambio".

Asimismo compartimos con Braslavsky (1999:20) que "numerosas investigaciones ofrecieron evidencia acerca de que la unidad más apropiada para la promoción de los cambios educativos tiene que ser la escuela. Sin embargo, todavía (...) no hay unanimidad de criterios" de cómo hacerlo.

Consideramos que entre otros desafíos que enfrenta actualmente nuestro sistema educativo público, se encuentra: (i) poder conceptualizar y focalizar aquellos tópicos que por ser multidimensionales, resultan medulares para el proceso de implementación de la reforma y (ii) definir las estrategias de intervención globales unas, específicas otras, que permitan consolidar los avances y trabajar las dificultades detectadas, tanto en las oficinas centrales como en las distintas unidades educativas.

Entendemos que un abordaje oportuno en este sentido, permitirá transformar potenciales amenazas en oportunidades, logrando así avances significativos en los procesos.

\section{2) Posibles alternativas de abordaje}

Consideremos ahora algunas estrategias que podrían permitir mayor articulación entre los distintos sectores implicados. Partiremos definiendo tres niveles desde los cuales pensar dicha integración:

1) político: eje rector de las distintas actividades que se desarrollan dentro del sistema y que se explicitan a través de objetivos que orientan la política educativa. Se relaciona con los sectores jerárquicos de cada uno de los escenarios que integran el sistema.

Resulta fundamental detectar cuáles son las principales líneas de acción que se establecen y cuáles las prioridades que se determinan en cada sector. Constatar si existen acuerdos o contradicciones entre los distintos ámbitos del sistema. Determinar los estilos de liderazgo que prevalecen; el clima de trabajo que se genera; las expectativas que existen en torno a los demás integrantes del plantel educativo.

Estos aspectos que ofician como marco institucional, ponen de manifiesto la mayor o menor coherencia interna que las unidades educativas y el sistema en su conjunto han logrado desarrollar, en el marco del proceso de cambio. Es fundamental tenerlos en cuenta, como estrategia para lograr una visión integradora de la realidad educativa en estudio.

2) personal - grupal: resulta fundamental considerar en cada escenario de intervención a las personas y a los grupos involucrados, tratando de evitar colisiones entre intereses institucionales, grupales e individuales.

También es importante considerar el rol que estas personas ocupan en los grupos y tener en cuenta el lugar que cada grupo les otorga (de líder positivo o negativo, de chivo expiatorio y segregado). Por otra parte, en este nivel resulta relevante definir las características de los lazos que unen a las personas y los grupos; (si están basados en la tarea, en rutinas administrativas y en aspectos afectivos). 
Desde un abordaje interactivo, será fundamental detectar e incorporar en las estrategias de intervención, a quienes ofician como referentes grupales o "nodos" de una eventual red. Nos referimos a las personas que integran varios grupos y establecen múltiples vínculos, teniendo la capacidad potencial de consolidar redes de colaboración.

3) cultural: la cultura institucional representa el componente que sostiene y articula aspectos personales y colectivos del escenario institucional, definiendo un cierto clima de trabajo y un determinado perfil de funcionamiento que prevalece en el centro.

Si se desconoce este nivel, se ignora de cierta forma el sustrato que oficia de plataforma de la dinámica cotidiana de cada unidad educativa y de cada sector del sistema.

Desde este punto de vista es importante decodificar los supuestos de base que llevan a que las personas actúen como lo hacen y no de otra manera; los mitos o las historias que están detrás de sus rutinas, las expectativas y los temores. Cuando se logran cambios en la matriz cultural, decimos que se ha conseguido institucionalizar un proceso de transformación.

Estos tres niveles estarían representando líneas de abordaje desde donde establecer estrategias de intervención globales, que permitan trabajar los ejes tanto en el ámbito de los centros como del sistema en su conjunto.

\section{3) Posibles estrategias para fortalecer la gestión}

¿Qué abordajes de análisis e intervención se deberían promover para fortalecer la gestión desde el punto de vista global e institucional? ¿Qué acciones podrían consolidar estos procesos?

A continuación haremos un punteo de posibles estrategias cuya implementación podría contribuir a fortalecer la gestión, superando los ejes de tensión.

\section{A nivel de políticas educativas}

1. Se deberían asegurar las condiciones necesarias que permitan consolidar nuevas modalidades de trabajo, a partir de estrategias diferenciales que den apoyo a las transformaciones en marcha, de acuerdo a la etapa de desarrollo en la que cada sector se encuentra. Estas estrategias deberían pensarse no sólo para los centros, sino también para las propias oficinas centrales que componen el sistema de educación media.

Habitualmente los apoyos que se brindan en cuanto a logística, recursos, personal, equipamientos, son muy importantes en el momento de iniciar los procesos de transformación, aunque relativamente breves en el tiempo. Algunos centros pueden continuar implementando la transformación sin mayores dificultades, por contar con fortalezas en materia de la gestión. Otros en cambio, vuelven a formas anteriores de trabajo. Es importante poder pasar de políticas de impacto a otras de desarrollo sostenible, Vázquez (2001). 
2. Las experiencias piloto con las que se inician la mayor parte de las reformas educativas, requieren, luego estrategias que permitan su generalización. Estos nuevos escenarios globales de trabajo no siempre están previstos desde el inicio, lo que lleva luego a improvisaciones que terminan desvirtuando la propuesta original (ruptura de paquetes horarios, estructuras de la coordinación y perfil de funciones). Resulta vital aprender a trabajar en función de escenarios prospectivos que integren en sus proyecciones las diferentes etapas, y que permitan definir acciones específicas de soporte y acompañamiento para cada fase.

3. Los esfuerzos de apoyo y asesoramiento tienden a focalizarse en los centros, perdiendo de vista al sistema en su conjunto como estructura integradora. Desde un punto de vista estratégico, es necesario definir líneas de acción que promuevan la integración y que involucren activamente a los distintos sectores que componen el sistema educativo.

\section{A nivel personal y grupal}

1. Es importante mantener un cierto equilibrio entre lo personal y lo grupal. Si pensamos en los grupos como potenciales unidades operativas, resulta relevante establecer estrategias para que estos colectivos se transformen en verdaderos equipos de trabajo.

2. Es necesario trabajar la diversidad. La heterogeneidad del alumnado y del plantel docente cada vez es mayor en cuanto a necesidades, a historias de vida, a demandas de apoyaturas, etc. Este fenómeno requiere de buenos estrategas, a la hora de establecer prioridades, atender demandas, cambiar rutinas, sin perder de vista el objetivo principal de la escuela, que es el de educar.

3. Ni el sistema educativo ni los centros de enseñanza pueden volverse organizaciones asistencialistas que funcionen en torno a las múltiples demandas que cada contexto realiza. En este aspecto, la estrategia a fortalecer tal vez sea la de "aprender a trabajar con otros", integrando redes de colaboración con otras instituciones de la comunidad, que atiendan necesidades específicas a partir de estrategias y competencias también específicas. Ello permitiría quitar presión a las escuelas y centrar su esfuerzo en educar.

\section{A nivel de cultura}

1. Es fundamental abordar desde este ángulo los diferentes "ejes de tensión" detectados, como los presentados en el diagrama. Asimismo, es importante establecer los supuestos de base que sustentan las propuestas, atendiendo la percepción que los actores involucrados tienen de la realidad analizada. El estudio de casos, es una metodología que ha demostrado ser de gran utilidad a la hora de intentar decodificar las lógicas que sustentan las prácticas colectivas, Vázquez (2003).

2. Por último, algo que Lidia Fernández (1999) denomina "destejer relaciones" y que refiere al análisis de los estilos con que las personas (administrativo, docentes, estudiantes y personal de servicio) abordan habitualmente las tareas que tienen encomendadas. Esta línea de análisis, permite al gestor institucional detectar posibles vicios de funcionamiento, que pueden resultar de enorme relevancia para comprender las lógicas instituidas en cada sector del sistema. 
Los múltiples niveles de abordaje que debe enfrentar la gestión, a partir de estrategias de intervención específicas para cada caso, nos llevan a entender esta labor como una tarea de claro perfil artesanal, enclavada sin embargo en procesos estandarizados de cambio. De allí el enorme desafío que, a nuestro entender, implica articular en el sistema educativo en su conjunto, procesos de fortalecimiento institucional con otros de generalización de reforma educativa.

\section{Bibliografía}

Braslavsky, C. 1999. Re-haciendo escuelas. Hacia un nuevo paradigma en la educación latinoamericana. Buenos Aires: Santillana.

Braslavsky, C. 2001. La dinámica del cambio educativo para el siglo XXI: acerca de la emergencia del paradigma en red. (Foro) IIPE. Buenos Aires: Fundación Ford.

Brunner, J. 2000. Educación: Escenarios de Futuro. PREAL.

Casassus, J. 2002. Problemas de la gestión educativa en América Latina. UNESCO

Fernández, L. 1999. Abordajes institucionales en la investigación del fenómeno educativo. En: Revista Escuela y Educación. Año VIII, Nº 14. Buenos Aires: UBA.

Hargreaves, A. 2001. Aprender a cambiar. Barcelona: Octaedro.

Marchesi, A. y Martín, E. 1998. Calidad de la enseñanza en tiempos de cambio. Madrid: Alianza Editores.

Vázquez, M.I. 2001. Armando el puzzle de la transformación institucional. (Material de circulación interna). Consejo de educación Secundaria: ANEP.

Vázquez, M.I. 2001. De las políticas a las prácticas cotidianas. En: Cuadernos de Investigación Educativa, Vol 1, № 8. Montevideo: Universidad ORT Uruguay.

Vázquez, MI. 2003. La metodología de casos en el ámbito de la gestión Educativa. (Informe de Investigación). Instituto de Educación: Universidad ORT Uruguay.

* Master en Investigación Educativa, CIEP-IDRC, Canadá. Psicóloga. Consultora en gestión educativa. Jefa, Departamento de Investigación de Planeamiento Educativo, Consejo de Enseñanza Secundaria. Docente de la Maestría en Sociedad e Instituciones, Universidad Nacional de San Luis, Argentina. Coordinadora Académica, Diploma en Planificación y Gestión Educativa, Instituto de Educación. 